\title{
Projective dimension and symmetric algebra of graph ideals
}

\author{
Maurizio Imbesi
}




\title{
PROJECTIVE DIMENSION AND SYMMETRIC ALGEBRA OF GRAPH IDEALS
}

\author{
MAURIZIO IMBESI
}

Received 10 January, 2012

\begin{abstract}
In this note lower bounds for the projective dimension of edge ideals are determined and the integrality of the symmetric algebra of these ideals is studied for some classes of simple graphs.

Criterions about torsion freeness of the symmetric powers are also analyzed and conditions about the acyclicity of the Z-complex of edge ideals of graphs are obtained.
\end{abstract}

2000 Mathematics Subject Classification: 05C99; 15A78; 16E05

Keywords: edge ideals, projective dimension, symmetric algebra, complexes

\section{INTRODUCTION AND PRELIMINARIES}

Algebraically speaking, the edge ideals $I(\mathscr{E})$ of finite simple graphs $\mathscr{E}$ are generated by square-free monomials of degree 2 in the polynomial ring $R=k\left[X_{1}, \ldots, X_{n}\right]$, where $k$ is a field and $n$ the number of vertices of $\mathscr{E}$.

The present paper deals with important problems about the projective dimension and the symmetric algebra of $I(\mathscr{E})$, and the acyclicity of the Z-complex of $I(\mathscr{E})$ is also discussed. It is structured as follows.

Section 2 analyzes the projective dimension of the edge ideals of simple graphs with respect to the number of their edges and consequences of it.

In section 3 the symmetric algebra of the edge ideals of simple graphs $S_{R}(I(\mathscr{E}))$ is studied, bearing also in mind essential results in $[2,11]$, and it is shown when such an algebra is an integral domain.

In section 4 criterions about torsion freeness of the symmetric powers of $S_{R}(I(\mathscr{G}))$ are considered and acyclicity conditions for the complex $\mathcal{Z}(I(\mathscr{E}))$ are examined.

Let $\mathscr{E}$ be a graph, $V(\mathscr{E}), E(\mathscr{G})$ the sets of its vertices and edges, resp. $\mathscr{E}$ is said to be simple if, for all $\left\{v_{i}, v_{j}\right\} \in E(\mathscr{E}), i \neq j$, it is $v_{i} \neq v_{j}$.

A cycle of length $n, C_{n} \subset \mathcal{G}$, is an alternating sequence of $n+1$ distinct vertices and $n$ edges beginning and ending at the same vertex, in which each edge is incident to the two vertices immediately preceding and following it.

A tree is a connected graph without cycles. 
The degree of a vertex $v_{\ell} \in V(\mathcal{G})$, denoted by $\operatorname{deg}\left(v_{\ell}\right)$, is the number of edges incident with $v_{\ell}$, i.e. $\operatorname{deg}\left(v_{\ell}\right)=\max \left\{r \mid \exists v_{1}, \ldots, v_{r} \in V(\mathscr{E})\right.$ with $\left\{v_{i}, v_{\ell}\right\} \in E(\mathscr{E})$, $i=1, \ldots, r\}$. When $\operatorname{deg}\left(v_{\ell}\right)=0$, the vertex $v_{\ell}$ is said isolated.

A subset $A$ of $V(\mathscr{E})$ is said a minimal vertex cover for $\mathscr{H}$ if every edge of $\mathscr{E}$ is incident with one vertex in $A$ and there is no proper subset of $A$ having such property. If $A$ satisfies only the incident condition, $A$ is called a vertex cover for $\mathscr{E}$.

The smallest number of vertices in any minimal vertex cover for $\mathscr{E}$ is called vertex covering number of $\mathscr{G}$ and denoted by $\alpha_{0}(\mathscr{G})$.

If $V(\mathscr{G})=\left\{v_{1}, \ldots, v_{n}\right\}$ and $R=k\left[X_{1}, \ldots, X_{n}\right]$ is the polynomial ring over a field $k$ such that each variable $X_{i}$ corresponds to the vertex $v_{i}$, the edge ideal $I(\mathscr{E})$ associated to $\mathscr{G}$ is the ideal $\left(\left\{X_{i} X_{j} \mid\left\{v_{i}, v_{j}\right\} \in E(\mathscr{E})\right\}\right) \subset R$.

Note that the non-zero edge ideals are those generated by square-free monomials of degree 2. This implies that $I(\mathscr{E})$ is a graded ideal of $R$ of initial degree 2, that is $I(\mathscr{G})=\oplus_{i \geqslant 2}\left(I(\mathscr{E})_{i}\right)$.

If $E(\mathscr{G})=\varnothing$, i.e. $\mathscr{G}$ has isolated vertices only, then $I(\mathscr{E})=(0)$.

So $I(\mathscr{G})$ has a graded free resolution of length at most $n$. The length of the (unique) minimal resolution of $I(\mathscr{G})$ is equal to $\operatorname{pdim}_{R}(I(\mathscr{E}))$, the projective dimension of $I(\mathcal{G})$.

An ideal $\wp \subset R$ generated by $A=\left\{X_{i_{1}}, \ldots, X_{i_{r}}\right\}$ is a minimal prime ideal of $I(\mathscr{G})$ if and only if $A$ is a minimal vertex cover for $\mathscr{E}$.

It is well-known that $\mathrm{ht}(I(\mathscr{G}))=\alpha_{0}(\mathscr{G})$ ([14], Corollary 6.1.18).

$\mathscr{E}$ is said a Cohen-Macaulay graph over $k(C-M$ for short $)$ if $\operatorname{depth}(R / I(\mathscr{E}))=$ $\operatorname{dim}(R / I(\mathscr{E}))$, where $\operatorname{dim}$ is the Krull dimension.

If $\mathscr{G}$ is a C-M graph, the Auslander and Buchsbaum theorem ([4], Corollary 3.3) gives $\operatorname{pdim}_{R}(I(\mathscr{G}))=\alpha_{0}(\mathscr{E})-1$.

The first initial Betti number in a minimal graded resolution of $I(\mathscr{E})$ has the following geometric interpretation (see [3]).

Proposition 1. If $\mathcal{E}$ is a graph with edge ideal $I(\mathcal{E})$ and

$$
\begin{gathered}
0 \longrightarrow \bigoplus_{i=1}^{c_{g}} R^{b_{g_{i}}}\left(-d_{g_{i}}\right) \longrightarrow \ldots \longrightarrow R^{c}(-4) \oplus R^{b}(-3) \longrightarrow \\
\longrightarrow R^{|E(\mathscr{E})|}(-2) \longrightarrow I(\mathscr{E}) \longrightarrow 0
\end{gathered}
$$

is the minimal graded resolution of $I(\mathscr{E})$, then $b=|E(\mathscr{L}(\mathscr{G}))|-N_{t}$, being $N_{t}$ the number of triangles in $\mathcal{E}$, and $\mathscr{L}(\mathcal{E})$ the edge graph of $\mathcal{E}$.

Remark 1. The number $c$ in a minimal graded resolution of $I(\mathscr{E})$ is equal to the number of unordered pairs of lines $f_{i}, f_{j}$ such that $f_{i}$ and $f_{j}$ are independent and cannot be joined by an edge.

Let $I$ be an ideal of $R=k\left[X_{1}, \ldots, X_{n}\right]$. The symmetric algebra of $I$ over $R$ is

$$
S_{R}(I)=\bigoplus_{t \geqslant 0} S_{t}(I)
$$


Let $R^{m} \stackrel{\varphi}{\longrightarrow} R^{q} \stackrel{\psi}{\longrightarrow} I \longrightarrow 0$ be a presentation of $I$, where $\varphi=\left(a_{i j}\right)$ is an $q \times m$ matrix with entries in $R$. Then

$$
S_{R}(I) \simeq R\left[T_{1}, \ldots, T_{q}\right] / J,
$$

being $J=\left(f_{1}, \ldots, f_{m}\right)$ the ideal of relations of $S_{R}(I), f_{j}=\sum_{i, j} a_{i j} T_{i}$.

For each $p=0, \ldots, n$ let's consider the morphism

$$
\partial_{p}: \bigwedge^{p} R^{n} \longrightarrow \bigwedge^{p-1} R^{n} \otimes I
$$

defined by

$$
\begin{aligned}
& \qquad \partial_{p}\left(e_{i_{1}} \wedge \ldots \wedge e_{i_{p}}\right)=\sum_{j=1}^{p}(-1)^{j-1} e_{i_{1}} \wedge \ldots \wedge \widehat{e}_{i_{j}} \wedge \ldots \wedge e_{i_{p}} \otimes \psi\left(e_{i_{j}}\right) \\
& \text { where } e_{1}, \ldots, e_{n} \text { is a basis of } R^{n} \text { and } \widehat{e} \text { means omission. }
\end{aligned}
$$

Let's denote $Z_{p}(I)=\operatorname{ker} \partial_{p}$.

Then one can build the complex:

$$
\begin{gathered}
Z(I): 0 \longrightarrow Z_{n}(I) \otimes S[-n] \stackrel{\partial_{n}^{\prime}}{\longrightarrow} Z_{n-1}(I) \otimes S[-n+1] \longrightarrow \ldots \\
\quad \ldots \longrightarrow Z_{1}(I) \otimes S[-1] \stackrel{\partial_{1}^{\prime}}{\longrightarrow} Z_{0}(I) \otimes S \longrightarrow S_{R}(I) \longrightarrow 0
\end{gathered}
$$

where $\partial_{p}^{\prime}$ is the map induced by $\partial_{p}$, and $S[-p]_{t}=S_{t-p}$. More information about the complexes $\mathcal{Z}(I)$ can be found in [6].

\section{BOUNDS FOR PROJECTIVE DIMENSION}

In this section we find lower bounds for the projective dimension of the edge ideals of finite simple graphs with respect to the number of their edges.

Theorem 1. Let $I(\mathscr{E})$ be the edge ideal of a simple graph $\mathscr{E}$ with $|E(\mathscr{E})| \geqslant 4$. Then $\operatorname{pdim}_{R}(I(\mathscr{E}))>1$.

Proof. It results $\operatorname{pdim}_{R}(I(\mathscr{E})) \geqslant \alpha_{0}(\mathscr{E})-1$.

So when $\alpha_{0}(\mathscr{E}) \geqslant 3$, then $\operatorname{pdim}_{R}(I(\mathscr{G}))>1$ and we conclude.

We have to show only the cases $\alpha_{0}(\mathscr{E})=1,2$.

$\alpha_{0}(\mathscr{G})=1$.

There is a unique graph $\mathscr{E}_{0}=\left\{v, v_{1}, v_{2}, v_{3}, v_{4}\right\}$ with $\left|E\left(\mathscr{E}_{0}\right)\right|=4$.

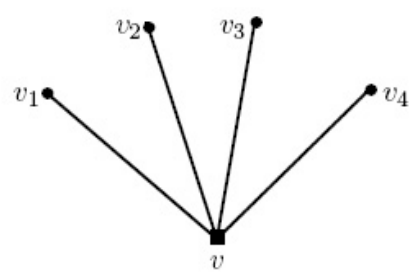


Every other graph $\mathscr{Y}_{0}^{\prime}$ with $\left|E\left(\mathscr{G}_{0}^{\prime}\right)\right|>4$ must contain $\mathscr{Y}_{0}$ and it is obtained by adding to $\mathscr{E}_{0} \ell$ distinct edges of initial vertex $v$.

The graded minimal resolution of $I\left(\mathscr{E}_{0}\right)$ is

$$
0 \rightarrow R(-5) \rightarrow R^{4}(-4) \rightarrow R^{6}(-3) \stackrel{\varphi}{\longrightarrow} R^{4}(-2) \rightarrow I\left(\mathscr{E}_{0}\right) \rightarrow 0 ;
$$

it results $\operatorname{pdim}_{R}\left(I\left(\mathscr{E}_{0}\right)\right)=3>1$. The graded minimal resolution of $I\left(\mathscr{G}_{0}^{\prime}\right)$ will be

$$
\begin{aligned}
0 & \rightarrow R(-5-\ell) \rightarrow R^{\left(\begin{array}{c}
4+\ell \\
4+\ell-1
\end{array}\right)(-4-\ell)} \rightarrow \ldots \\
\ldots & \rightarrow R^{\left(\begin{array}{c}
4+\ell \\
2
\end{array}\right)}(-3) \rightarrow R^{4+\ell}(-2) \rightarrow I\left(\mathcal{G}_{0}^{\prime}\right) \rightarrow 0 .
\end{aligned}
$$

Then $\operatorname{pdim}_{R}\left(I\left(\mathscr{G}_{0}^{\prime}\right)\right)>\operatorname{pdim}_{R}\left(I\left(\mathscr{E}_{0}\right)\right)>1$.

$$
\alpha_{0}(\mathscr{G})=2 \text {. }
$$

We have to consider only the following simple graphs, each having 4 edges:

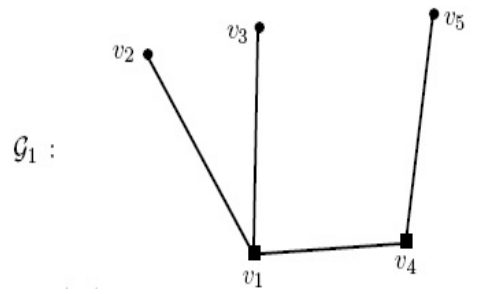

Resolution of $I\left(\mathscr{E}_{1}\right)$ :

$$
0 \rightarrow R(-4) \rightarrow R^{4}(-3) \stackrel{\varphi}{\longrightarrow} R^{4}(-2) \rightarrow I\left(\mathscr{E}_{1}\right) \rightarrow 0 .
$$

$\mathcal{G}_{2}$ :

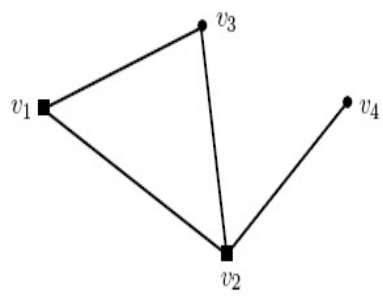

Resolution of $I\left(\mathscr{G}_{2}\right)$ :

$$
0 \rightarrow R(-4) \rightarrow R^{4}(-3) \stackrel{\varphi}{\longrightarrow} R^{4}(-2) \rightarrow I\left(\mathscr{E}_{2}\right) \rightarrow 0 .
$$

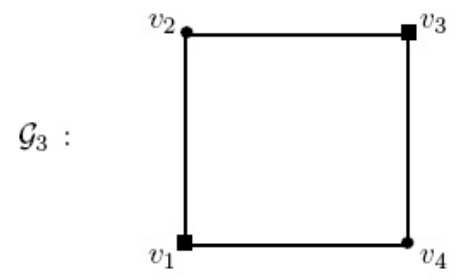


Resolution of $I\left(\mathscr{G}_{3}\right)$ :

$$
0 \rightarrow R(-4) \rightarrow R^{4}(-3) \stackrel{\varphi}{\longrightarrow} R^{4}(-2) \rightarrow I\left(\mathscr{E}_{3}\right) \rightarrow 0 .
$$

$\mathcal{G}_{4}$

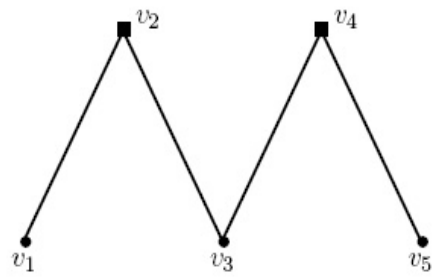

Resolution of $I\left(\mathscr{E}_{4}\right)$ :

$$
0 \rightarrow R(-5) \rightarrow R^{3}(-3) \oplus R(-4) \stackrel{\varphi}{\longrightarrow} R^{4}(-2) \rightarrow I\left(\mathcal{E}_{4}\right) \rightarrow 0 .
$$

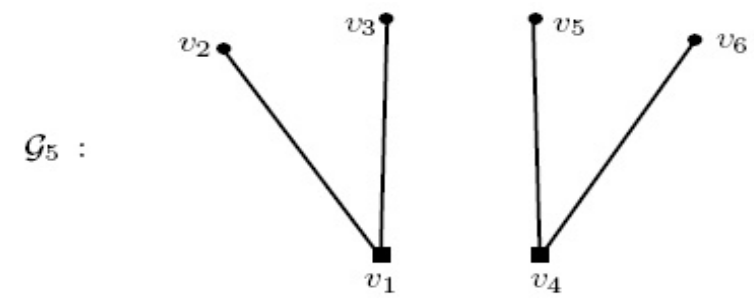

Resolution of $I\left(\mathscr{E}_{5}\right)$ :

$0 \rightarrow R(-6) \rightarrow R^{4}(-5) \rightarrow R^{2}(-3) \oplus R^{4}(-4) \stackrel{\varphi}{\longrightarrow} R^{4}(-2) \rightarrow I\left(\mathscr{E}_{5}\right) \rightarrow 0$.

It results $\operatorname{pdim}_{R}\left(I\left(\mathscr{G}_{i}\right)\right)=2$, for $i=1,2,3,4 ; \operatorname{pdim}_{R}\left(I\left(\mathscr{G}_{5}\right)\right)=3$.

If we add to any of the above graphs $\mathscr{E}_{i}, i=1,2,3,4$, a finite number of distinct edges such that in the new graphs $\mathscr{H}_{i}^{\prime}, i=1,2,3,4, \alpha_{0}\left(\mathscr{H}_{i}^{\prime}\right)=\alpha_{0}\left(\mathscr{E}_{i}\right)$, we have, by Proposition 1, that $b^{\prime}+c^{\prime} \geqslant b+c$, where $b^{\prime}$ and $c^{\prime}$ are the graded Betti numbers of the first syzygy module in the resolution of $I\left(\mathscr{G}_{i}^{\prime}\right)$.

Because $\operatorname{pdim}_{R}\left(I\left(\mathscr{G}_{i}\right)\right)=2$, then $\operatorname{pdim}_{R}\left(I\left(\mathscr{G}_{i}^{\prime}\right)\right)>1$.

Similar arguments hold for $\mathscr{E}_{5}$, except in the following situation:

let's $\mathscr{E}_{5}^{\prime}$ be the graph obtained from $\mathscr{S}_{5}$ by adding to it the edge $\left\{v_{1}, v_{4}\right\}$. Unlike the other cases, the edge ideal of $\mathscr{E}_{5}^{\prime}$ has projective dimension strictly less than $\operatorname{pdim}_{R}\left(I\left(\mathscr{E}_{5}\right)\right)$.

In fact, the resolution of $I\left(\mathscr{G}_{5}^{\prime}\right)$ is :

$$
0 \rightarrow R^{2}(-4) \rightarrow R^{6}(-3) \rightarrow R^{5}(-2) \rightarrow I\left(\mathscr{E}_{5}^{\prime}\right) \rightarrow 0 .
$$

However $\operatorname{pdim}_{R}\left(I\left(\mathscr{E}_{5}^{\prime}\right)\right)=2>1$.

It is possible to improve the above lower bound.

Proposition 2. Let $\mathscr{E}$ be as in Theorem 1. Let $\alpha(\mathscr{E})$ be the size of the largest minimal vertex cover for $\mathscr{E}$. Then $\operatorname{pdim}_{R}(I(\mathscr{E})) \geqslant \alpha(\mathscr{E})-1$. 
Proof. Note that $\operatorname{pdim}_{R}(I(\mathscr{E}))=\operatorname{reg}\left(R / I(\mathscr{E})^{\wedge}\right)$, the regularity of $R / I(\mathscr{E})^{\wedge}$, where $I(\mathscr{G})^{\wedge}$ is the Alexander dual of $I(\mathscr{E})$.

Because the Alexander dual is generated by the minimal vertex covers for $\mathcal{E}$, the degree of its largest generator is $\alpha(\mathscr{G})$.

So $\operatorname{reg}\left(R / I(\mathscr{G})^{\wedge}\right) \geqslant \alpha(\mathscr{G})-1$.

For example, let's consider the graph $\mathscr{E}_{0}$ that appears in the proof of Theorem 1. We see that any graph $\mathscr{E}$ that contains $\mathscr{E}_{0}$ as an induced subgraph will have at least one minimal vertex cover of at least size 4 . In fact, the minimal vertex cover that does not contain $v$ must contain $v_{1}, v_{2}, v_{3}, v_{4}$. So $\operatorname{pdim}_{R}(I(\mathscr{E})) \geqslant 3$.

Corollary 1. The only simple graphs with projective dimension of their edge ideal less than or equal to 1 are the following:

$\mathcal{P}:=\left\{v_{1}, v_{2}\right\}$, an edge.

$\mathcal{Q}:=\left\{\left\{v_{1}, v_{2}, v_{3}\right\},\left\{\left\{v_{1}, v_{2}\right\},\left\{v_{2}, v_{3}\right\}\right\}\right\}$, two consecutive edges.

$\mathcal{R}:=\left\{\left\{v_{1}, v_{2}, v_{3}, v_{4}\right\},\left\{\left\{v_{1}, v_{2}\right\},\left\{v_{3}, v_{4}\right\}\right\}\right\}$, two non consecutive edges.

$\mathcal{s}:=\left\{\left\{v_{1}, v_{2}, v_{3}\right\},\left\{\left\{v_{1}, v_{2}\right\},\left\{v_{2}, v_{3}\right\},\left\{v_{1}, v_{3}\right\}\right\}\right\}$, a triangle.

$\mathcal{T}:=\left\{\left\{v_{1}, v_{2}, v_{3}, v_{4}\right\},\left\{\left\{v_{1}, v_{2}\right\},\left\{v_{2}, v_{3}\right\},\left\{v_{3}, v_{4}\right\}\right\}\right\}$, three consecutive edges.

\section{INTEGRALITY FOR SYMMETRIC ALGEBRA}

In this section we examine meaningful classes of simple graphs and study the integrality of the symmetric algebra of their edge ideal.

Proposition 3. Let $I(\mathscr{E})$ be the edge ideal of a simple graph $\mathscr{E}$ with $|E(\mathscr{E})|<4$. Then the symmetric algebra $S_{R}(I(\mathscr{E}))$ is an integral domain.

Proof. Let's consider every simple graph with at most 3 edges and construct explicitly the symmetric algebra of its edge ideal.

- Graph with 2 nodes and 1 edge

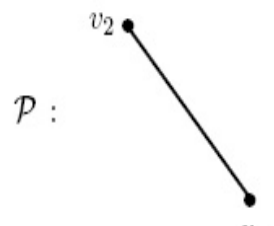

Resolution of $I(\mathcal{P})$ :

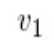

$0 \rightarrow R(-2) \rightarrow I(\mathcal{P}) \rightarrow 0$

It is $S_{R}(I(\mathcal{P}))=R[Y] / J, J=(0)$; obviously, $S_{R}(I(\mathcal{P}))$ is integral.

- Graph with 3 nodes and 2 edges 


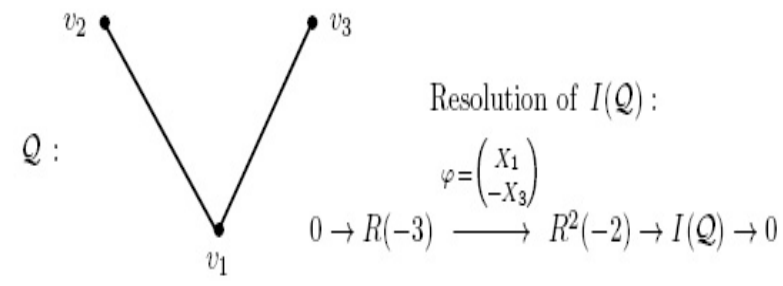

$S_{R}(I(Q))=R\left[Y_{1}, Y_{2}\right] / J, \quad J=\left(X_{1} Y_{1}-X_{3} Y_{2}\right)$.

$J \subset k\left[X_{1}, X_{2}, X_{3}, Y_{1}, Y_{2}\right]$ is prime, so $S_{R}(I(\mathcal{Q}))$ is integral.

- Graph with 4 nodes and 2 edges

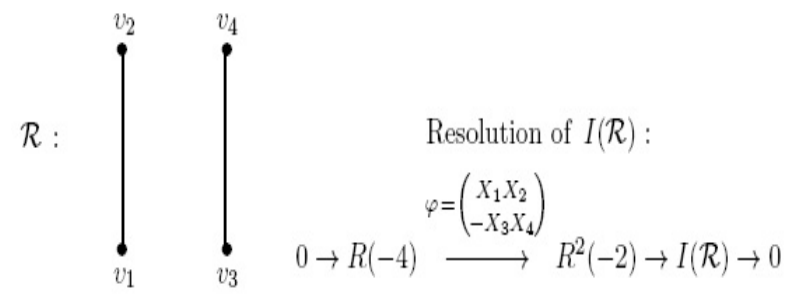

$S_{R}(I(\mathcal{R}))=R\left[Y_{1}, Y_{2}\right] / J, \quad J=\left(X_{1} X_{2} Y_{1}-X_{3} X_{4} Y_{2}\right)$.

$J \subset k\left[X_{1}, X_{2}, X_{3}, X_{4}, Y_{1}, Y_{2}\right]$ is prime, so $S_{R}(I(\mathcal{R}))$ is integral.

- Graph with 3 nodes and 3 edges

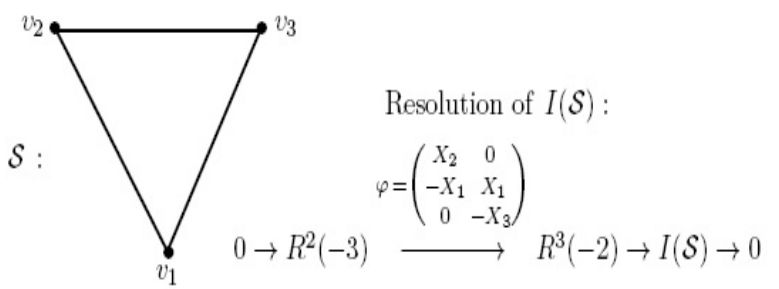

$S_{R}(I(8))=R\left[Y_{1}, Y_{2}, Y_{3}\right] / J, \quad J=\left(X_{2} Y_{1}-X_{1} Y_{2}, X_{1} Y_{2}-X_{3} Y_{3}\right)$ $J \subset k\left[X_{1}, X_{2}, X_{3}, Y_{1}, Y_{2}, Y_{3}\right]$ is prime, so $S_{R}(I(\&))$ is integral.

- Graph (tree) with 4 nodes and 3 edges

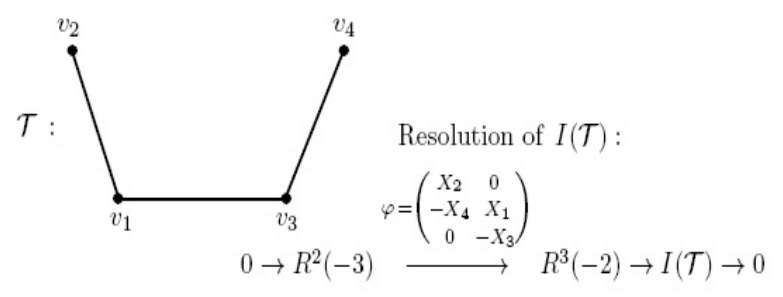

$S_{R}(I(\mathcal{T}))=R\left[Y_{1}, Y_{2}, Y_{3}\right] / J, \quad J=\left(X_{2} Y_{1}-X_{4} Y_{2}, X_{1} Y_{2}-X_{3} Y_{3}\right)$. $J \subset k\left[X_{1}, \ldots, X_{4}, Y_{1}, Y_{2}, Y_{3}\right]$ is prime, so $S_{R}(I(\mathcal{T}))$ is integral.

- Graph with 4 nodes and 3 edges 


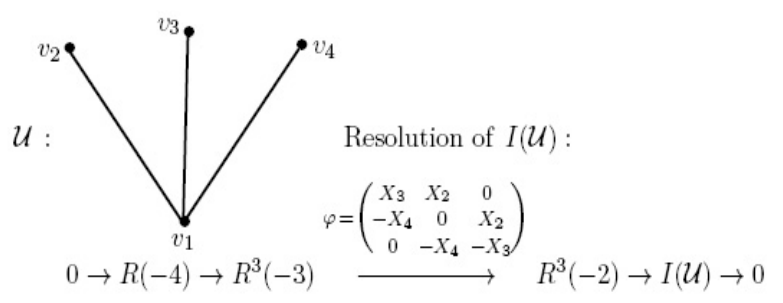

$S_{R}(I(\mathcal{U}))=R\left[Y_{1}, Y_{2}, Y_{3}\right] / J$, where $J=\left(X_{3} Y_{1}-X_{4} Y_{2}, X_{2} Y_{1}-X_{4} Y_{3}, X_{2} Y_{2}-X_{3} Y_{3}\right)$.

$J \subset k\left[X_{1}, \ldots, X_{4}, Y_{1}, Y_{2}, Y_{3}\right]$ is prime, so $S_{R}(I(U))$ is integral.

- Graph with 5 nodes and 3 edges

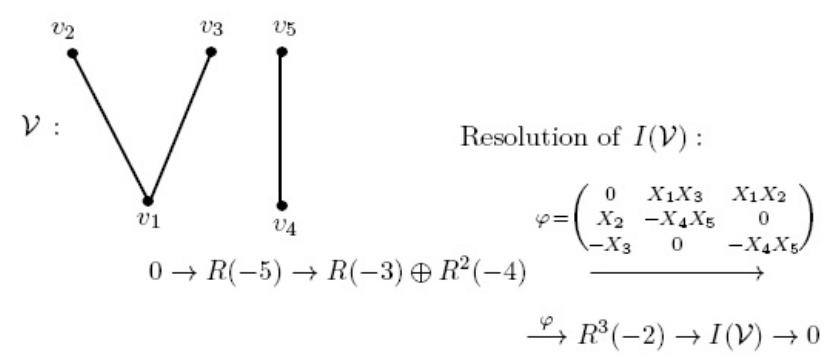

$S_{R}(I(\mathcal{V}))=R\left[Y_{1}, Y_{2}, Y_{3}\right] / J$, where

$J=\left(X_{2} Y_{2}-X_{3} Y_{3}, X_{1} X_{3} Y_{1}-X_{4} X_{5} Y_{2}, X_{1} X_{2} Y_{1}-X_{4} X_{5} Y_{3}\right)$.

$J \subset k\left[X_{1}, \ldots, X_{5}, Y_{1}, Y_{2}, Y_{3}\right]$ is prime, so $S_{R}(I(\mathcal{V}))$ is integral.

- Graph with 6 nodes and 3 edges

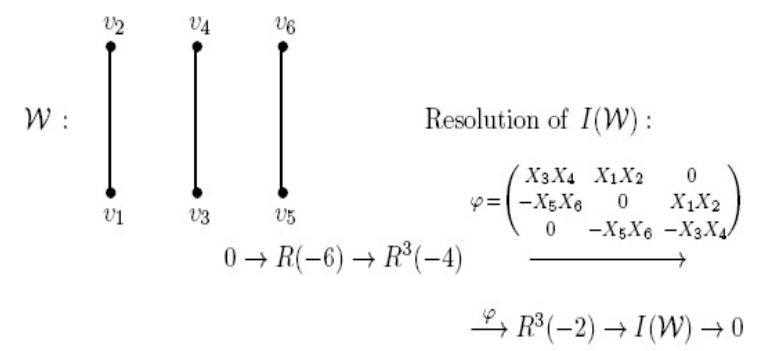

$S_{R}(I(\mathcal{W}))=R\left[Y_{1}, Y_{2}, Y_{3}\right] / J$, where

$J=\left(X_{3} X_{4} Y_{1}-X_{5} X_{6} Y_{2}, X_{1} X_{2} Y_{1}-X_{5} X_{6} Y_{3}, X_{1} X_{2} Y_{2}-X_{3} X_{4} Y_{3}\right)$.

$J \subset k\left[X_{1}, \ldots, X_{6}, Y_{1}, Y_{2}, Y_{3}\right]$ is prime, so $S_{R}(I(\mathcal{W}))$ is integral.

Corollary 2. Let $\mathscr{E}$ be a simple graph such that $\operatorname{pdim}_{R}(I(\mathscr{E})) \leqslant 1$. Then $S_{R}(I(\mathscr{E}))$ is an integral domain.

Proof. By Corollary 1, the result immediately descends. 
Let $\mathcal{E}$ be a Cohen-Macaulay graph with $q$ edges. By [14], Exercise 6.2.19, if $g$ is the height of $I(\mathscr{E})$, it results $q \leqslant\left(g^{2}+g\right) / 2$. Hence the possible C-M graphs with $\alpha_{0}(\mathscr{E})=3$ have at most 6 edges.

Proposition 4. Let $\&$ be a Cohen-Macaulay graph with no cycles of even length and $\operatorname{pdim}_{R}(I(\mathscr{E}))=2$. Then the symmetric algebra $S_{R}(I(\mathscr{E}))$ is an integral domain.

Proof. Remember that for C-M graphs it results $\operatorname{pdim}_{R}(I)=\alpha_{0}-1$.

By Proposition 3, the symmetric algebra of edge ideals of generic simple graphs having at most 3 edges is an integral domain.

So it remains to examine the symmetric algebra of edge ideals of C-M graphs $\mathcal{E}$ with $\operatorname{pdim}_{R}(I(\mathscr{G}))=2$ and $4 \leqslant|E(\mathscr{E})| \leqslant 6$.

Computations are made using softwares COCOA [13] and Macaulay [5].

* C-M graphs with $\operatorname{pdim}_{R}(I)=2$ having 4 edges are the following:

$\mathscr{H}_{1}$

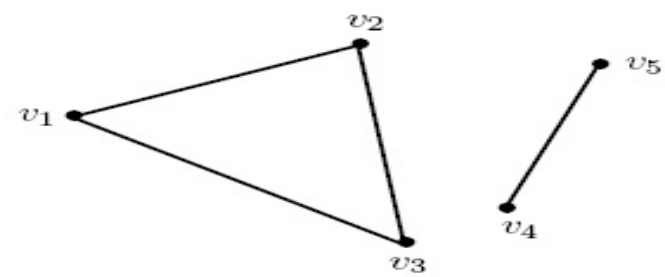

The minimal free resolution of its edge ideal is

$$
\begin{gathered}
0 \rightarrow R^{2}(-5) \rightarrow R^{2}(-3) \oplus R^{3}(-4) \stackrel{\varphi}{\longrightarrow} R^{4}(-2) \rightarrow I\left(\mathcal{H}_{1}\right) \rightarrow 0 . \\
S_{R}\left(I\left(\mathscr{H}_{1}\right)\right)=R\left[Y_{1}, \ldots, Y_{4}\right] / J, \text { where } \\
J=\left(X_{1} Y_{3}-X_{3} Y_{4}, X_{2} Y_{2}-X_{3} Y_{4}, X_{2} X_{3} Y_{1}-X_{4} X_{5} Y_{3},\right. \\
\left.X_{1} X_{3} Y_{1}-X_{4} X_{5} Y_{2}, X_{1} X_{2} Y_{1}-X_{4} X_{5} Y_{4}\right) . \\
J \subset k\left[X_{1}, \ldots, X_{5}, Y_{1}, \ldots, Y_{4}\right] \text { is prime, so } S_{R}\left(I\left(\mathscr{H}_{1}\right)\right) \text { is integral. }
\end{gathered}
$$

$\mathscr{H}_{2}$

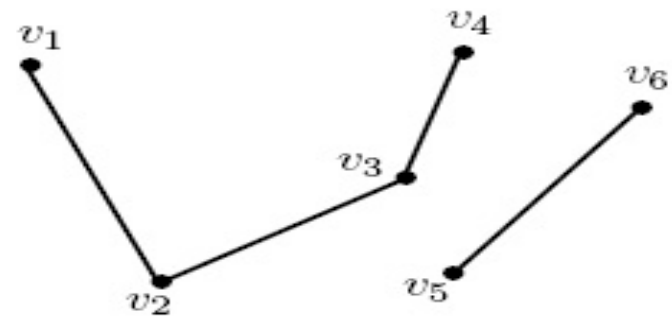

The ideal of relations of $S_{R}\left(I\left(\mathscr{H}_{2}\right)\right)$ is

$$
J=\left(X_{2} Y_{2}-X_{4} Y_{3}, X_{1} Y_{3}-X_{3} Y_{4}, X_{3} X_{4} Y_{1}-X_{5} X_{6} Y_{2},\right.
$$




$$
\left.X_{2} X_{3} Y_{1}-X_{5} X_{6} Y_{3}, X_{1} X_{2} Y_{1}-X_{5} X_{6} Y_{4}\right) .
$$

$J \subset k\left[X_{1}, \ldots, X_{6}, Y_{1}, \ldots, Y_{4}\right]$ is prime, so $S_{R}\left(I\left(\mathscr{H}_{2}\right)\right)$ is integral.

* C-M graphs with $\operatorname{pdim}_{R}(I)=2$ having 5 edges are the following: $\mathscr{H}_{3}$

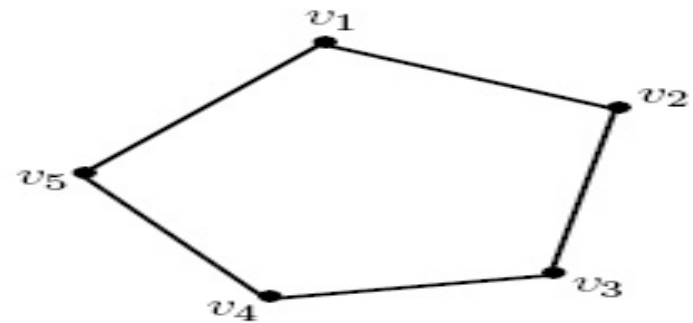

The minimal free resolution of its edge ideal is

$$
0 \rightarrow R(-5) \rightarrow R^{5}(-3) \stackrel{\varphi}{\longrightarrow} R^{5}(-2) \rightarrow I\left(\mathscr{H}_{3}\right) \rightarrow 0 .
$$

The ideal of relations of $S_{R}\left(I\left(\mathscr{H}_{3}\right)\right)$ is

$$
\begin{aligned}
J= & \left(X_{3} Y_{2}-X_{5} Y_{3}, X_{4} Y_{1}-X_{1} Y_{2}, X_{2} Y_{1}-X_{5} Y_{5},\right. \\
& \left.X_{2} Y_{3}-X_{4} Y_{4}, X_{1} Y_{4}-X_{3} Y_{5}\right) .
\end{aligned}
$$

$J \subset k\left[X_{1}, \ldots, X_{5}, Y_{1}, \ldots, Y_{5}\right]$ is prime, so $S_{R}\left(I\left(\mathscr{H}_{3}\right)\right)$ is integral.

$\mathscr{H}_{4}$

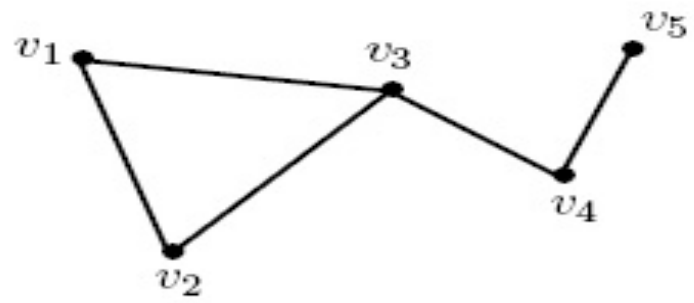

The minimal free resolution of its edge ideal is

$$
\begin{aligned}
& 0 \rightarrow R(-4) \oplus R(-5) \rightarrow R^{5}(-3) \oplus R(-4) \stackrel{\varphi}{\longrightarrow} R^{5}(-2) \rightarrow I\left(\mathscr{H}_{4}\right) \rightarrow 0 . \\
& J=\left(X_{3} Y_{1}-X_{5} Y_{2}, X_{2} Y_{2}-X_{4} Y_{4}, X_{1} Y_{2}-X_{4} Y_{3}, X_{2} Y_{3}-X_{1} Y_{4},\right. \\
&\left.X_{1} Y_{4}-X_{3} Y_{5}, X_{1} X_{2} Y_{1}-X_{4} X_{5} Y_{5}\right) \text { is a prime ideal. }
\end{aligned}
$$

So the symmetric algebra $S_{R}\left(I\left(\mathscr{H}_{4}\right)\right)$ is integral. 
$\mathscr{H}_{5}$

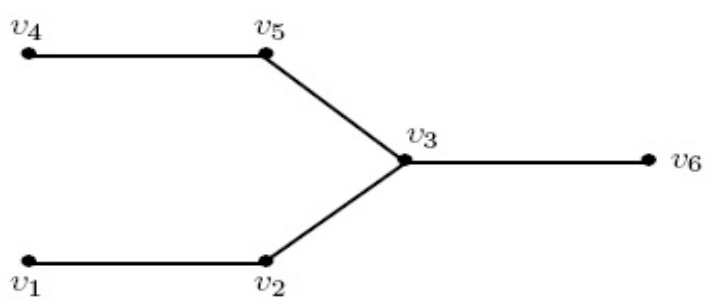

$$
\begin{aligned}
J= & \left(X_{6} Y_{1}-X_{5} Y_{3}, X_{2} Y_{3}-X_{6} Y_{4}, X_{4} Y_{1}-X_{3} Y_{2}, X_{2} Y_{1}-X_{5} Y_{4},\right. \\
& \left.X_{1} Y_{4}-X_{3} Y_{5}, X_{1} X_{2} Y_{2}-X_{4} X_{5} Y_{5}\right) \text { is a prime ideal. }
\end{aligned}
$$

So the symmetric algebra $S_{R}\left(I\left(\mathscr{H}_{5}\right)\right)$ is integral.

* C-M graphs with $\operatorname{pdim}_{R}(I)=2$ having 6 edges are the following: $\mathscr{H}_{6}$

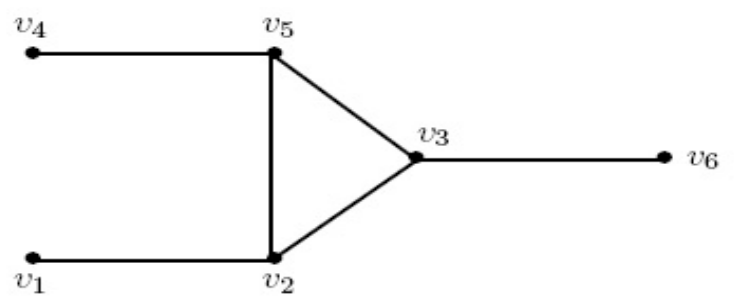

The minimal free resolution of its edge ideal is

$$
0 \rightarrow R^{3}(-4) \rightarrow R^{8}(-3) \stackrel{\varphi}{\longrightarrow} R^{6}(-2) \rightarrow I\left(\mathscr{H}_{6}\right) \rightarrow 0 .
$$

The ideal of relations of $S_{R}\left(I\left(\mathscr{H}_{6}\right)\right)$ is

$$
\begin{aligned}
J= & \left(X_{6} Y_{1}-X_{5} Y_{4}, X_{2} Y_{4}-X_{6} Y_{5}, X_{4} Y_{1}-X_{3} Y_{3}, X_{4} Y_{2}-X_{2} Y_{3},\right. \\
& \left.X_{2} Y_{1}-X_{3} Y_{2}, X_{3} Y_{2}-X_{5} Y_{5}, X_{1} Y_{2}-X_{5} Y_{6}, X_{1} Y_{5}-X_{3} Y_{6}\right) .
\end{aligned}
$$

$J \subset k\left[X_{1}, \ldots, X_{6}, Y_{1}, \ldots, Y_{6}\right]$ is prime, so $S_{R}\left(I\left(\mathscr{H}_{6}\right)\right)$ is integral.

$\mathscr{H}_{7}$

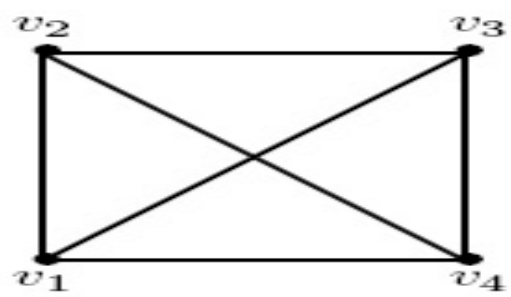

The ideal of relations of $S_{R}\left(I\left(\mathscr{H}_{7}\right)\right)$ is

$$
J=\left(X_{3} Y_{1}-X_{2} Y_{4}, X_{2} Y_{4}-X_{4} Y_{5}, X_{4} Y_{2}-X_{3} Y_{3}, X_{3} Y_{3}-X_{1} Y_{4},\right.
$$


$\left.X_{1} Y_{1}-X_{2} Y_{3}, X_{2} Y_{3}-X_{4} Y_{6}, X_{2} Y_{2}-X_{1} Y_{5}, X_{1} Y_{5}-X_{3} Y_{6}\right)$.

$J$ is not a prime ideal, so $S_{R}\left(I\left(\mathscr{H}_{7}\right)\right)$ is not integral.

$\mathscr{H}_{8}$

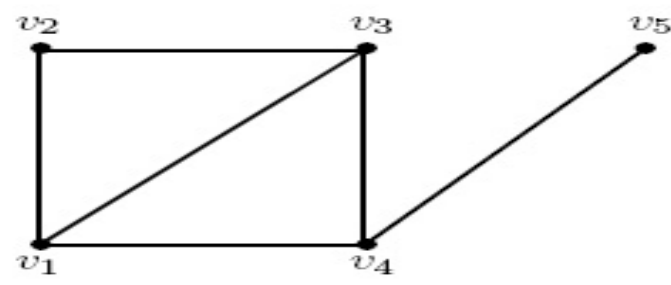

$$
\begin{aligned}
J= & \left(X_{3} Y_{1}-X_{5} Y_{4}, X_{1} Y_{1}-X_{5} Y_{3}, X_{2} Y_{4}-X_{4} Y_{5}, X_{4} Y_{2}-X_{3} Y_{3},\right. \\
& \left.X_{3} Y_{3}-X_{1} Y_{4}, X_{2} Y_{3}-X_{4} Y_{6}, X_{2} Y_{2}-X_{1} Y_{5}, X_{1} Y_{5}-X_{3} Y_{6}\right) .
\end{aligned}
$$

$J$ is not a prime ideal, so $S_{R}\left(I\left(\mathscr{H}_{8}\right)\right)$ is not integral.

$\mathscr{H}_{9}$

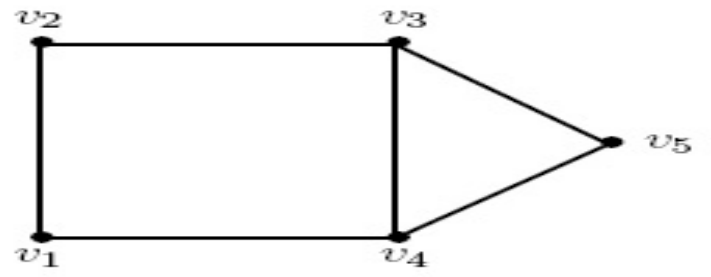

$$
J=\left(X_{3} Y_{1}-X_{4} Y_{2}, X_{4} Y_{2}-X_{5} Y_{4}, X_{1} Y_{1}-X_{5} Y_{3}, X_{2} Y_{2}-X_{5} Y_{5},\right.
$$$$
\left.X_{2} Y_{4}-X_{4} Y_{5}, X_{3} Y_{3}-X_{1} Y_{4}, X_{2} Y_{3}-X_{4} Y_{6}, X_{1} Y_{5}-X_{3} Y_{6}\right) \text {. }
$$

$J$ is not a prime ideal, so $S_{R}\left(I\left(\mathscr{H}_{9}\right)\right)$ is not integral.

$\mathscr{H}_{10}$

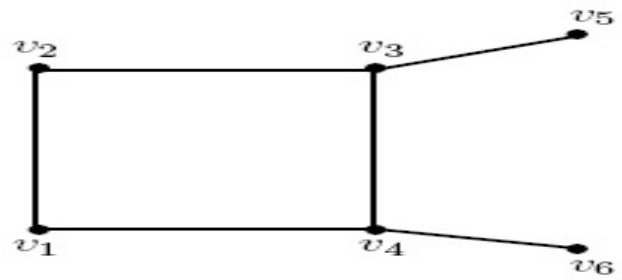

$$
\begin{aligned}
J= & \left(X_{3} Y_{1}-X_{6} Y_{4}, X_{1} Y_{1}-X_{6} Y_{3}, X_{4} Y_{2}-X_{5} Y_{4}, X_{2} Y_{2}-X_{5} Y_{5},\right. \\
& \left.X_{2} Y_{4}-X_{4} Y_{5}, X_{3} Y_{3}-X_{1} Y_{4}, X_{2} Y_{3}-X_{4} Y_{6}, X_{1} Y_{5}-X_{3} Y_{6}\right) .
\end{aligned}
$$

$J$ is not a prime ideal, so $S_{R}\left(I\left(\mathscr{H}_{10}\right)\right)$ is not integral.

Proposition 4 can be easily extended as follows. 
Theorem 2. Let $\mathscr{E}$ be a simple graph such that $|E(\mathscr{E})| \leqslant 6$, with no cycles of even length, and $\alpha_{0}(\mathscr{E})=3$. Then the symmetric algebra $S(I(\mathscr{E}))$ is an integral domain.

In the light of previous results, we are able to show that the symmetric algebra is integral for the following classes of simple graphs.

1) Connected graphs of the type

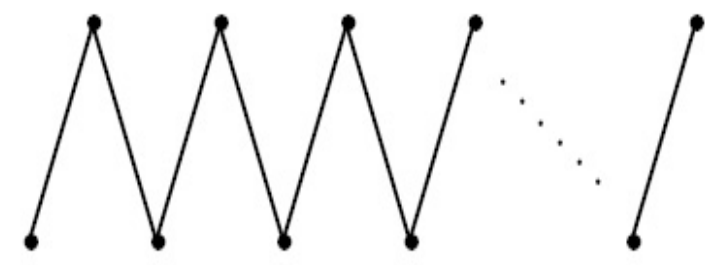

2) Connected graphs (star graphs) of the type

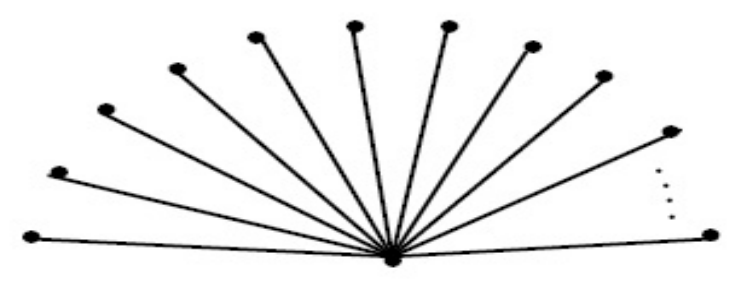

3) Non-connected graphs of the type

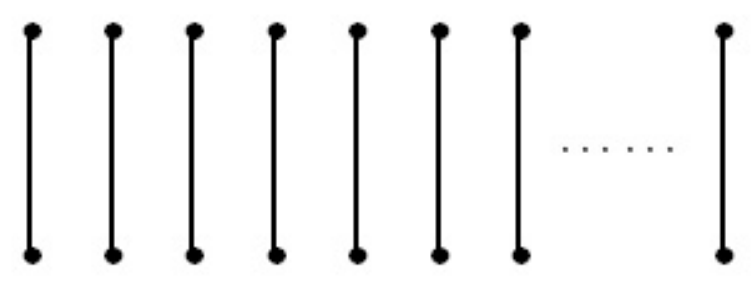

Theorem 3. Let $\mathcal{G}$ be a simple graph as in items 1) - 3). Then the symmetric algebra $S(I(\mathscr{E}))$ is an integral domain .

Proof. The statement is shown for star graphs (item 2)) with $n+1$ vertices and $n$ edges.

Let $R=k\left[X_{1}, \ldots, X_{n+1}\right]$ be the polynomial ring whose variables are associated to the vertices of $\mathscr{E}$. The edge ideal of $\mathscr{E}$ is $I(\mathscr{E})=\left(X_{1} X_{n+1}, \ldots, X_{n} X_{n+1}\right)$. 
The minimal free resolution of $I(\mathscr{E})$ is

$$
\begin{gathered}
0 \rightarrow R(-n-1) \rightarrow R^{\left(\begin{array}{c}
n \\
n-1
\end{array}\right)}(-n) \rightarrow R^{\left(\begin{array}{c}
n \\
n-2
\end{array}\right)}(-n+1) \rightarrow \ldots \\
\ldots \rightarrow R^{\left(\begin{array}{c}
n \\
3
\end{array}\right)}(-4) \rightarrow R^{\left(\begin{array}{c}
n \\
2
\end{array}\right)}(-3) \rightarrow R^{n}(-2) \rightarrow I(\mathscr{E}) \rightarrow 0 .
\end{gathered}
$$

The ideal of relations of $S_{R}(I(\mathcal{E}))$ is $J=\left(g_{1}, g_{2}, \ldots, g_{\left(\begin{array}{c}n \\ 2\end{array}\right)}\right)$, where

$$
\begin{array}{ccc}
g_{1}=X_{n-1} Y_{1}-X_{n} Y_{2} & & \\
g_{2}=X_{n-2} Y_{1}-X_{n} Y_{3} & g_{n}=X_{n-2} Y_{2}-X_{n-1} Y_{3} & \\
g_{3}=X_{n-3} Y_{1}-X_{n} Y_{4} & g_{n+1}=X_{n-3} Y_{2}-X_{n-1} Y_{4} & g_{2 n-2}=X_{n-3} Y_{3}-X_{n-2} Y_{4} \\
g_{4}=X_{n-4} Y_{1}-X_{n} Y_{5} & g_{n+2}=X_{n-4} Y_{2}-X_{n-1} Y_{5} & g_{2 n-1}=X_{n-4} Y_{3}-X_{n-2} Y_{5} \\
\ldots \ldots & \ldots \cdots & \cdots \cdots \\
g_{n-1}=X_{1} Y_{1}-X_{n} Y_{n} & g_{2 n-3}=X_{1} Y_{2}-X_{n-1} Y_{n} & g_{3 n-6}=X_{1} Y_{3}-X_{n-2} Y_{n} \\
& & \\
\ldots \ldots \ldots & g_{\left(\begin{array}{c}
n \\
2
\end{array}\right)-2}=X_{2} Y_{n-2}-X_{3} Y_{n-1} & \\
& g_{\left(\begin{array}{c}
n \\
2
\end{array}\right)-1}=X_{1} Y_{n-2}-X_{3} Y_{n} & g_{\left(\begin{array}{c}
n \\
2
\end{array}\right)}=X_{1} Y_{n-1}-X_{2} Y_{n} .
\end{array}
$$

For all $n \in \mathbb{N}, J$ is a prime ideal, so $S_{R}(I(\mathscr{E}))$ is integral.

\section{INTEGRALITY AND ACYCLICITY}

In this section we study conditions about the acyclicity of the Z-complex of edge ideals and join them with integrality.

Remark 2. In 1964 Micali (cfr. [7]) showed, when $R$ is an integral domain and $E$ a finitely generated $R$-module, that the symmetric algebra $S_{R}(E)$ is an integral domain if and only if $S_{t}(E)$ is torsion free, $\forall t$.

So, for all simple graphs examined in last section, the symmetric powers $S_{t}(I(\mathscr{E}))$ are torsion free, $\forall t$.

In particular, for simple graphs such that $\operatorname{pdim}_{R}(I(\mathscr{E}))=1$ (Corollaries 1 and 2), $S_{t}(I(\mathscr{G}))$ torsion free is equivalent to the inequality grade $I_{k}(\varphi) \geqslant|E(\mathscr{E})|-1-k+1$, for all $k=1, \ldots, \operatorname{rank}(\varphi)$ (see [1]).

The following joins integrality and acyclicity.

Proposition 5. Let $\mathcal{G}$ be one of the graphs as in Corollary 1. The complex $\mathcal{Z}\left(S_{t}(I(\mathscr{E}))\right)$ is acyclic if and only if the symmetric algebra $S_{R}\left(S_{t}(I(\mathcal{E}))\right)$ is an integral domain.

Proof. By Corollary 2, $S_{R}(I(\mathscr{E}))$ is an integral domain.

By Micali's theorem (cfr. Remark 2), $S_{t}(I(\mathscr{E}))$ is torsion-free, $\forall t$. Because $S_{t}(I(\mathscr{E}))$ is an $R$-module, $S_{R}\left(S_{t}(I(\mathscr{G}))\right)$ integral domain is equivalent to be $\mathcal{Z}\left(S_{t}(I(\mathscr{E}))\right)$ acyclic, according to a remark of [10].

Remark 3. By [12], Remark 3.8 c), if $\mathscr{G}$ is a graph such that $S_{t}(I(\mathscr{G}))$ is torsion free, then the complex $\mathcal{Z}(I(\mathscr{E}))$ is exact if and only if $\mathcal{Z}(I(\mathscr{G}))_{\wp}$ is exact for any $\wp \in \operatorname{Spec}(R)$ with depth $R_{\wp}<|E(\mathscr{E})|$. 
Proposition 6. Let $\mathscr{E}$ be a graph with $|E(\mathscr{E})| \geqslant 4$. Then $S_{R}(I(\mathscr{E}))$ is not a complete intersection.

Proof. By [12], Theorem 2.9, $S_{R}(I(\mathscr{E}))$ is a complete intersection if and only if $\operatorname{pdim}_{R}(I(\mathscr{G})) \leqslant 1$.

But when $|E(\mathscr{G})| \geqslant 4$, Proposition 1 says that $\operatorname{pdim}_{R}(I(\mathscr{E}))>1$.

So $S_{R}(I(\mathscr{E}))$ is not a complete intersection.

Proposition 7. The edge ideals of graphs $\mathscr{E}$ with $\operatorname{pdim}_{R}(I(\mathscr{E}))=2$ and rank $N=$ $\operatorname{rank} Z_{1}(I(\mathscr{G}))=3$ have the $Z$-complex acyclic.

Proof. $\operatorname{rank} N=3 \Longrightarrow\left(\bigwedge^{3} N\right)^{* *} \simeq R$ and the Z-complex is

$$
\begin{gathered}
\mathcal{Z}(I(\mathscr{E})): 0 \rightarrow R \otimes S[-3] \rightarrow\left(\wedge^{2} N\right)^{* *} \otimes S[-2] \rightarrow \\
\quad \rightarrow N \otimes S[-1] \rightarrow R^{q} \otimes S \rightarrow S_{R}(I(\mathscr{E})) \rightarrow 0
\end{gathered}
$$

Because $I(\mathscr{G})$ is torsion-free, $\left(\bigwedge^{2} N\right)^{* *} \simeq Z_{2}(I(\mathscr{G}))($ in fact, $2<\operatorname{rank} N)$ and $Z_{2}(I(\mathscr{G}))$ is a reflexive module.

It follows that $\left(\bigwedge^{2} N\right)^{* *}$ is a reflexive module. Such a module is a second syzygy module, so depth $\left(\bigwedge^{2} N\right)^{* *} \geqslant 2$ (see $[4,8]$ ).

¿From Peskine and Szpiro's acyclicity lemma (see [9], Lemma 1.8), the complex $\mathcal{Z}(I(\mathscr{G}))$ is exact.

Corollary 3. The only $C-M$ graphs with $\operatorname{pdim}_{R}(I)=2$ and such that rank $Z_{1}(I)$ $=3$ are the following:

1) $\mathscr{H}_{1}:=\left\{\left\{v_{1}, v_{2}, v_{3}, v_{4}, v_{5}\right\},\left\{\left\{v_{1}, v_{2}\right\},\left\{v_{2}, v_{3}\right\},\left\{v_{1}, v_{3}\right\},\left\{v_{4}, v_{5}\right\}\right\}\right\}$, a triangle + an isolated edge.

2) $\mathscr{H}_{2}:=\left\{\left\{v_{1}, v_{2}, v_{3}, v_{4}, v_{5}, v_{6}\right\},\left\{\left\{v_{1}, v_{2}\right\},\left\{v_{2}, v_{3}\right\},\left\{v_{3}, v_{4}\right\},\left\{v_{5}, v_{6}\right\}\right\}\right\}$, three consecutive edges + an isolated edge.

Proof. Looking at the minimal free resolutions of the edge ideals of $\mathscr{H}_{1}$ and $\mathscr{H}_{2}$ as in Proposition 4, it results that $\operatorname{rank} Z_{1}\left(I\left(\mathscr{H}_{1}\right)\right)$ and rank $Z_{1}\left(I\left(\mathscr{H}_{2}\right)\right)$ are both equal to $3=\operatorname{rank} \varphi$.

Note that, by Proposition 7, the complexes $\mathcal{Z}\left(I\left(\mathscr{H}_{1}\right)\right)$ and $\mathcal{Z}\left(I\left(\mathscr{H}_{2}\right)\right)$ are acyclic.

\section{ACKNOWLEDGEMENT}

The author gratefully thanks Giovanni Molica Bisci for his useful suggestions and comments in drafting this article. 


\section{REFERENCES}

[1] L. L. Avramov, "Complete intersections and symmetric algebras," J. Algebra, vol. 73, pp. 248263, 1981.

[2] G. M. Bisci, "Symmetric algebra of ideals of graphs," in Stochastic geometry, convex bodies, empirical measures and applications to engineering science, ser. Suppl. Rend. Circ. Mat. Palermo, II. Ser. 70, R. Schneider, Ed., vol. II. Palermo: Circolo Matematico di Palermo, 2002, pp. 167175.

[3] S. Eliahou and R. H. Villarreal, "The second Betti number of an edge ideal," in Proceedings of 31th national congress of the Mexican Mathematical Society, Hermosillo, Mexico, October 11-17, 1999, P. Padilla, Ed. México: Sociedad Matemática Mexicana, 1999, pp. 115-119.

[4] E. Evans and P. Griffith, Syzygies, ser. London Mathematical Society Lecture Note Series. Cambridge: Cambridge University Press, 1985, vol. 106.

[5] D. Grayson and M. Stillman, Macaulay2: a software system for research in algebraic geometry. electronic, 1993-2002. [Online]. Available: http://math.uiuc.edu

[6] J. Herzog, A. Simis, and W. V. Vasconcelos, "On the arithmetic and homology of algebras of linear type," Trans. Am. Math. Soc., vol. 283, pp. 661-683, 1984.

[7] A. Micali, "Sur les algèbres universelles," Ann. Inst. Fourier, vol. 14, no. 2, pp. 33-88, 1964.

[8] G. Molica and G. Restuccia, "Torsion free exterior powers of a module and their resolutions," An. Ştiinţ. Univ. “Ovidius” Constanţa, Ser. Mat., vol. 10, no. 1, pp. 101-108, 2002.

[9] C. Peskine and L. Szpiro, "Dimension projective finie et cohomologie locale. applications à la demonstration de conjectures de M. Auslander, H. Bass et A. Grothendieck," Publ. Math., Inst. Hautes Étud. Sci., vol. 42, pp. 47-119, 1972.

[10] G. Restuccia, "Puissances symétriques sans torsion," Cahiers Mathém., Montpellier, vol. 39, 1992.

[11] G. Restuccia, "On the symmetric algebra for a module of projective dimension two," An. Univ. Bucur., Mat., vol. 40, no. 1-2, pp. 83-91, 1991.

[12] G. Restuccia and C. Ionescu, " $q$-torsion freeness of symmetric powers," Rend. Circ. Mat. Palermo, II. Ser., vol. 46, no. 3, pp. 329-346, 1997.

[13] L. Robbiano and CoCoATeam, CoCoA 4: a system for doing computations in commutative algebra. electronic, 2000. [Online]. Available: http://cocoa.dima.unige.it

[14] R. H. Villarreal, Monomial algebras, ser. Pure and Applied Mathematics. New York: Marcel Dekker, 2001, vol. 238.

Author's address

Maurizio Imbesi

University of Messina, Department of Mathematics, V.le Stagno d'Alcontres, 31, 98166 Messina, Italy

E-mail address: imbesim@unime.it 